\title{
Laserlike and atomlike regimes in a one-atom laser
}

\author{
A. D. Boozer \\ California Institute of Technology, Norman Bridge Laboratory of Physics 12-33, Pasadena, California 91125, USA
}

(Received 19 September 2008; published 10 November 2008)

\begin{abstract}
We consider a three-level model of a one-atom laser, and show that there are two limiting regimes of parameter space, one in which the system behaves like a conventional laser and one in which the system exhibits novel quantum properties. We show that in the first limiting regime, the model can be approximated by semiclassical laser theory, and in the second limiting regime the model can be approximated as an effective two-level atom. We also perform numerical simulations that confirm the limiting behaviors predicted by these approximate descriptions.
\end{abstract}

DOI: 10.1103/PhysRevA.78.053814

PACS number(s): 42.50.Pq

\section{INTRODUCTION}

There is both theoretical [1-12] and experimental [13] interest in lasers in which the gain medium consists of a single atom. In this paper, we consider a one-atom laser model consisting of a three-level atom coupled to a singlemode optical cavity, and show that there are two limiting regimes of parameter space, one in which the system behaves like a conventional laser and one in which the system exhibits novel quantum properties. Specifically, we identify a key parameter $N_{\gamma}$, the critical photon number, which can be thought of as the number of photons that must be present in the cavity in order to saturate the lasing transition of the atom. We show that the behavior of the system depends critically on the value of $N_{\gamma}$ : for sufficiently large values of $N_{\gamma}$, the system behaves like a conventional laser and can be approximated by semiclassical laser theory, and for sufficiently small values of $N_{\gamma}$ the system exhibits novel quantum properties and can be approximated as an effective two-level atom. We derive and analytically solve the semiclassical and two-level atom theories that apply in these limiting regimes, and we show how the two regimes are delineated. We also perform numerical simulations of the model, which confirm that its limiting behaviors are correctly accounted for by the semiclassical and two-level atom theories.

The paper is organized as follows. In Sec. II, we describe the one-atom laser model and write down the master equation that governs the evolution of the density matrix for the system. In Sec. III, we obtain a simplified description of the system by making a semiclassical approximation in which we neglect the correlations between products of quantum operators. We derive the equations of motion for the system in the semiclassical approximation, and find the steady-state solution to these equations. In Sec. IV, we return to the full quantum description of the system, and show that in the limit of large $N_{\gamma}$ the system is well described by the semiclassical theory derived in Sec. III. We perform computer simulations of the system to confirm this prediction, and show that as we decrease $N_{\gamma}$, the semiclassical theory begins to break down. In Sec. V, we show that in the limit of small $N_{\gamma}$, the system can be approximated by a different theory in which the system is treated as an effective two-level atom.

\section{ONE-ATOM LASER MODEL}

The model that we will be considering describes a threelevel atom that is coupled to a single-mode cavity and is driven by a classical field; similar models have been considered in $[1,2]$. A level diagram for the system is shown in Fig. 1. As shown in the diagram, the atom has internal states 1,2 , and 3 . State 3 spontaneously decays to state 2 at rate $\gamma$, state 2 spontaneously decays to state 1 at rate $\Gamma$, and the energy decay rate of the cavity is $\kappa$. The 2-3 transition is resonantly coupled to the cavity mode with coupling strength $g$, and the 1-3 transition is resonantly driven by a classical field with Rabi frequency $\Omega$. For simplicity, we will choose the phase of the classical field such that $\Omega$ is real.

The Hamiltonian for the coupled atom-cavity system is

$$
H=g\left(a \hat{A}_{32}+a^{\dagger} \hat{A}_{23}\right)+(\Omega / 2)\left(\hat{A}_{31}+\hat{A}_{13}\right),
$$

where $\hat{A}_{j k} \equiv|j\rangle\langle k|$ are atomic raising and lowering operators and $a^{\dagger}$ and $a$ create and annihilate photons in the cavity mode. The state of the system can be described in terms of a density matrix $\rho$, which evolves in time according to the master equation

$$
\dot{\rho}=-i[H, \rho]+\mathcal{L}_{12} \rho+\mathcal{L}_{23} \rho+\mathcal{L}_{c} \rho,
$$

where

$$
\mathcal{L}_{23} \rho=(\gamma / 2)\left(2 \hat{A}_{23} \rho \hat{A}_{32}-\hat{A}_{33} \rho-\rho \hat{A}_{33}\right),
$$

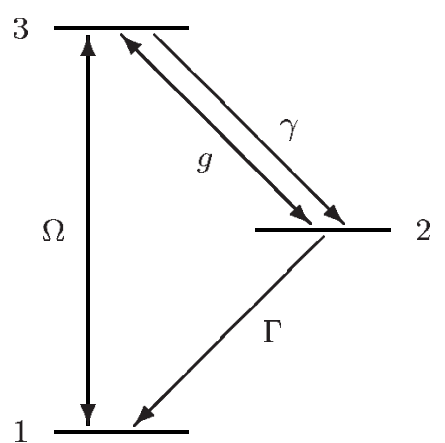

FIG. 1. Level diagram for the one-atom laser. The horizontal lines indicate the atomic states 1,2 , and 3 . The 1-3 transition is driven by a classical field with Rabi frequency $\Omega$, and the $2-3$ transition is coupled to an optical cavity with coupling strength $g$. State 3 spontaneously decays to state 2 at rate $\gamma$, and state 2 spontaneously decays to state 1 at rate $\Gamma$. 


$$
\begin{gathered}
\mathcal{L}_{12} \rho=(\Gamma / 2)\left(2 \hat{A}_{12} \rho \hat{A}_{21}-\hat{A}_{22} \rho-\rho \hat{A}_{22}\right), \\
\mathcal{L}_{c} \rho=(\kappa / 2)\left(2 a \rho a^{\dagger}-a^{\dagger} a \rho-\rho a^{\dagger} a\right)
\end{gathered}
$$

describe the spontaneous decay on the 2-3 transition, the spontaneous decay on the 1-2 transition, and the cavity decay.

Intuitively, we can think of the 2-3 transition as the lasing transition, and we can think of the classical field as the laser pump. Note that the decay from state 2 to state 1 draws population from state 2 , which in a conventional laser would maintain a population inversion across the lasing transition.

\section{SEMICLASSICAL THEORY}

\section{A. Semiclassical equations of motion}

We can gain some insight into the system by considering a semiclassical approximation in which we ignore correlations between products of quantum operators. This type of approximation is discussed in $[1,3,4]$. We first use the master equation (2) to derive equations of motion for the expectation values of the atomic operators $\hat{A}_{i j}$ and the photon annihilation operator $a$ :

$$
\begin{gathered}
\dot{A}_{11}=-i(\Omega / 2)\left(A_{13}-A_{31}\right)+\Gamma A_{22} \\
\dot{A}_{22}=-i g\left(\left\langle a^{\dagger} \hat{A}_{23}\right\rangle-\left\langle a \hat{A}_{32}\right\rangle\right)-\Gamma A_{22}+\gamma A_{33} \\
\dot{A}_{33}=-i g\left(\left\langle a \hat{A}_{32}\right\rangle-\left\langle a^{\dagger} \hat{A}_{23}\right\rangle\right)-i(\Omega / 2)\left(A_{31}-A_{13}\right)-\gamma A_{33}, \\
\dot{A}_{12}=-i g\left\langle a^{\dagger} \hat{A}_{13}\right\rangle+i(\Omega / 2) A_{32}-(\Gamma / 2) A_{12} \\
\dot{A}_{23}=-i g\left(\left\langle a \hat{A}_{22}\right\rangle-\left\langle a \hat{A}_{33}\right\rangle\right)-i(\Omega / 2) A_{21}-(1 / 2)(\Gamma+\gamma) A_{23} \\
\dot{A}_{31}=-i(\Omega / 2)\left(A_{33}-A_{11}\right)+i g\left\langle a^{\dagger} \hat{A}_{21}\right\rangle-(\gamma / 2) A_{31} \\
\dot{\alpha}=-i g A_{23}-(\kappa / 2) \alpha .
\end{gathered}
$$

Here $\langle\hat{X}\rangle \equiv \operatorname{Tr}[\rho \hat{X}]$ is the expectation value of $\hat{X}$, where $\hat{X}$ is an arbitrary operator, and $A_{i j} \equiv\left\langle\hat{A}_{i j}\right\rangle$ and $\alpha \equiv\langle a\rangle$ are the expectation values of the operators $\hat{A}_{i j}$ and $a$. These equations of motion do not form a closed system, because some of the equations depend on the expectation values of products of operators; for example, the equation of motion for $A_{22}$ depends on $\left\langle a \hat{A}_{32}\right\rangle$. Given two operators $\hat{X}$ and $\hat{Y}$, we can express the expectation value of their product as

$$
\langle\hat{X} \hat{Y}\rangle=\langle\hat{X}\rangle\langle\hat{Y}\rangle+C(\hat{X}, \hat{Y})
$$

where

$$
C(\hat{X}, \hat{Y})=\langle\hat{X} \hat{Y}\rangle-\langle\hat{X}\rangle\langle\hat{Y}\rangle
$$

describes the correlations between the two operators. We can obtain a closed set of equations by making a semiclassical approximation in which we neglect these correlations (an alternative method of deriving these equations is given in the Appendix):

$$
\begin{gathered}
\dot{A}_{11}=-i(\Omega / 2)\left(A_{13}-A_{31}\right)+\Gamma A_{22}, \\
\dot{A}_{22}=-i g\left(\alpha^{*} A_{23}-\alpha A_{32}\right)-\Gamma A_{22}+\gamma A_{33}, \\
\dot{A}_{33}=-i g\left(\alpha A_{32}-\alpha^{*} A_{23}\right)-i(\Omega / 2)\left(A_{31}-A_{13}\right)-\gamma A_{33}, \\
\dot{A}_{12}=-i g \alpha^{*} A_{13}+i(\Omega / 2) A_{32}-(\Gamma / 2) A_{12}, \\
\dot{A}_{23}=-i g \alpha\left(A_{22}-A_{33}\right)-i(\Omega / 2) A_{21}-(1 / 2)(\Gamma+\gamma) A_{23}, \\
\dot{A}_{31}=-i(\Omega / 2)\left(A_{33}-A_{11}\right)+i g \alpha^{*} A_{21}-(\gamma / 2) A_{31}, \\
\dot{\alpha}=-i g A_{23}-(\kappa / 2) \alpha .
\end{gathered}
$$

In Sec. IV, we will discuss the conditions under which the semiclassical approximation is justified, but for now let us investigate the theory that results when this approximation is made.

It is straightforward to check that the semiclassical equations of motion are invariant under the transformation

$$
\left(\alpha, A_{21}, A_{23}\right) \rightarrow\left(e^{i \phi} \alpha, e^{i \phi} A_{21}, e^{i \phi} A_{23}\right),
$$

where $\phi$ is an arbitrary phase. By exploiting this invariance, we can always choose $\alpha$ to be real; the equations of motion then imply that $A_{12}$ is real and that $A_{23}$ and $A_{31}$ are imaginary (note that $A_{11}, A_{22}$, and $A_{33}$ are always real, since they represent atomic populations). It is convenient to replace $A_{23}$ and $A_{31}$ with real-valued variables $B_{23}=-i A_{23}$ and $B_{31}=-i A_{31}$. In terms of the real-valued variables, the equations of motion are

$$
\begin{gathered}
\dot{A}_{11}=-\Omega B_{31}+\Gamma A_{22}, \\
\dot{A}_{22}=2 g \alpha B_{23}-\Gamma A_{22}+\gamma A_{33}, \\
\dot{A}_{33}=-2 g \alpha B_{23}+\Omega B_{31}-\gamma A_{33}, \\
\dot{A}_{12}=-g \alpha B_{31}+(\Omega / 2) B_{23}-(\Gamma / 2) A_{12}, \\
\dot{B}_{23}=-g \alpha\left(A_{22}-A_{33}\right)-(\Omega / 2) A_{12}-(1 / 2)(\Gamma+\gamma) B_{23}, \\
\dot{B}_{31}=-(\Omega / 2)\left(A_{33}-A_{11}\right)+g \alpha A_{12}-(\gamma / 2) B_{31}, \\
\dot{\alpha}=g B_{23}-(\kappa / 2) \alpha .
\end{gathered}
$$

\section{B. Steady-state solution}

Let us now investigate the steady-state solution to the semiclassical equations of motion (23)-(29). It is useful to 
first introduce some dimensionless parameters. We will define the critical atom number $N_{A}$ and the critical photon number $N_{\gamma}$ by

$$
N_{A}=\kappa \gamma / 4 g^{2}, \quad N_{\gamma}=\gamma^{2} / 4 g^{2} .
$$

Let us also define a scaled photon number $m$, a dimensionless pumping strength $p$, and a parameter $\eta$ that gives the ratio of the spontaneous decay rates for the 2-3 and 1-2 transitions:

$$
m=n / N_{\gamma}, \quad p=\Omega^{2} / \gamma \Gamma, \quad \eta=\gamma / \Gamma,
$$

where $n=|\alpha|^{2}$ is the intracavity photon number.

We can obtain the steady-state solution by setting Eqs. (23)-(29) to zero. From the equations $\dot{A}_{22}=0$ and $\dot{\alpha}=0$, we find that

$$
\kappa n+\gamma A_{33}=\Gamma A_{22} .
$$

We can understand this relation by noting that for every photon spontaneously emitted on the 1-2 transition, there is either one photon emitted from the cavity or one photon spontaneously emitted on the 2-3 transition. Thus, the sum of the rates for cavity emission and for spontaneous emission on the 2-3 transition must equal the rate for spontaneous emission on the 1-2 transition.

We can also solve the steady-state equations for the scaled photon number $m$; after some algebra, we find that either $m=0$ or $m$ is a solution to

$$
m^{2}+B(p) m+C(p)=0
$$

where

$$
\begin{gathered}
B(p)=p+1+2 / \eta, \\
C(p)=\left(1 / \eta^{2}\right)\left[(2+\eta) p^{2}+\left(2 \eta+2 / \eta+3-1 / N_{A}\right) p+1+\eta\right] .
\end{gathered}
$$

Note that $B(p)$ is always positive, but the sign of $C(p)$ depends on the value of $p$. If $C(p)>0$, then Eq. (33) has no physically relevant solutions and the steady-state scaled photon number is $m(p)=0$. If $C(p)<0$, then Eq. (33) has one physically relevant solution, which is given by

$$
m(p)=(1 / 2)\left[\sqrt{B^{2}(p)-4 C(p)}-B(p)\right] .
$$

One can show that if this solution exists it is stable, and the $m=0$ solution is unstable, so for $C(p)<0$ the steady-state scaled photon number is given by Eq. (36). Note that the steady-state scaled photon number depends only on the parameters $\eta$ and $N_{A}$; it is independent of $N_{\gamma}$.

The behavior of the system depends critically on the number of roots of $C(p)$, and this number is set by the parameters $\eta$ and $N_{A}$. Since $C(p)$ is quadratic, it can have zero roots, one root, or two roots. If $C(p)$ has zero roots, then $C(p)$ is always positive and $m(p)=0$ for all pumping strengths. In this case, we will say that the system is in the nonlasing regime. If $C(p)$ has two roots $p_{1}$ and $p_{2}$, then $m(p)=0$ for $p<p_{1}$ and $p>p_{2}$, and $m(p)$ is given by Eq. (36) for $p_{1}<p<p_{2}$. In this case, we will say that the system is in the lasing regime. The transition between the lasing and nonlasing regimes occurs when $C(p)$ has exactly one root, and this condition defines a

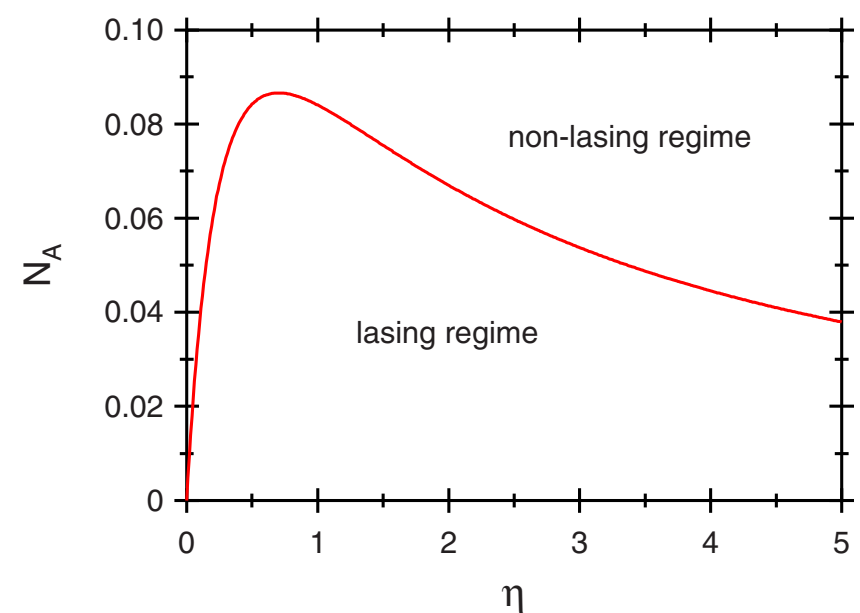

FIG. 2. (Color online) Lasing and nonlasing regimes of parameter space.

curve in the parameter space $\left(\eta, N_{A}\right)$ that is shown in Fig. 2. Above this curve, the system is in the nonlasing regime, and below the curve the system is in the lasing regime.

Let us assume that the system is in the lasing regime. The pumping strength $p_{1}$ defines the laser threshold. Below threshold $\left(p<p_{1}\right)$, the populations are

$$
\begin{gathered}
A_{11}=1-A_{22}-A_{33}, \\
A_{22}=\eta A_{33}, \\
A_{33}=(2+\eta+\eta / p)^{-1},
\end{gathered}
$$

and the atomic dipole moments are

$$
A_{12}=0, \quad B_{23}=0, \quad B_{31}=(\gamma / \Omega) A_{33} .
$$

Above threshold, for $p_{1}<p<p_{2}$, the populations are

$$
\begin{gathered}
A_{11}=1-A_{22}-A_{33}, \\
A_{22}=N_{A} \eta[m(p)+p+1+1 / \eta], \\
A_{33}=N_{A}(p+1+1 / \eta) .
\end{gathered}
$$

Note that as we increase the pumping strength $p$ beyond the laser threshold $p_{1}$, the laser output $m(p)$ reaches a maximum and then begins to decrease until it vanishes at $p=p_{2}$. This effect, known as self-quenching, is discussed in [3].

\section{FULL QUANTUM THEORY}

Let us now return to the full quantum theory described in Sec. II. The degree to which the full quantum theory agrees with the semiclassical theory depends on the value of the critical photon number $N_{\gamma}$. We can see this by considering the behavior of the system for pumping strengths near the semiclassical threshold $p_{1}$. For large values of $N_{\gamma}$, if the pumping strength is even slightly above the semiclassical threshold, then there are many photons in the cavity (recall that $n=N_{\gamma} m$ ), so the quantum fluctuations are small and the system is well described by the semiclassical theory. As we 


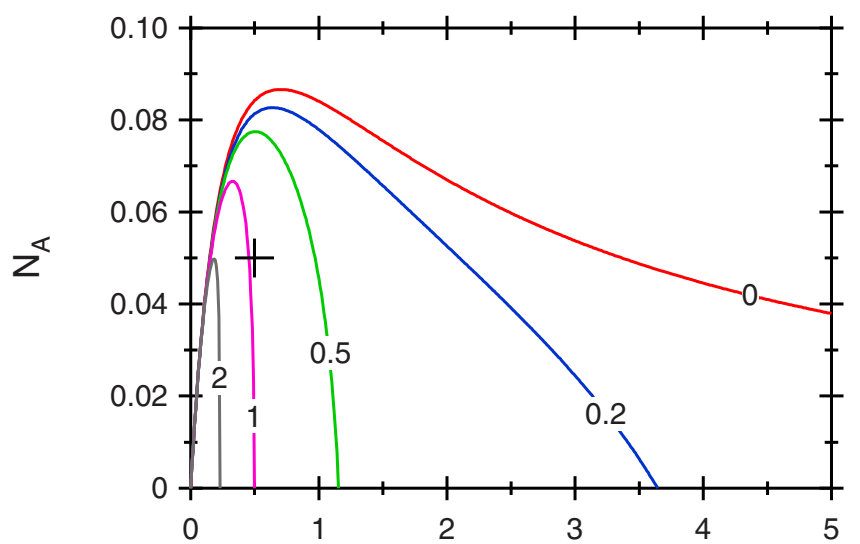

$\eta$

FIG. 3. (Color online) Lines of constant $m\left(2 p_{1}\right)$, as predicted by the semiclassical theory. Curves are shown for $m=0.0,0.2,0.5,1.0$, and 2.0. Note that the $m=0.0$ curve delineates the border between the semiclassical lasing and nonlasing regimes. The cross indicates the parameters $\eta=0.5, N_{A}=0.05$ that are used in the full quantum simulations.

reduce $N_{\gamma}$, there are fewer photons in the cavity and the semiclassical theory begins to break down. We can predict the values of $N_{\gamma}$ for which the semiclassical theory applies by using the semiclassical theory to calculate the scaled photon number at $2 p_{1}$ : when $m\left(2 p_{1}\right) \gg 1 / N_{\gamma}$ [which implies that $n\left(2 p_{1}\right) \gg 1$, we expect the system to be well approximated by the semiclassical theory. Figure 3 shows lines of constant $m\left(2 p_{1}\right)$ in the space of parameters $\left(\eta, N_{A}\right)$ that characterize the system; note that the $m=0$ curve delineates the lasing and nonlasing regimes. As we approach this curve from below, the value of $m\left(2 p_{2}\right)$ becomes smaller and smaller, so larger and larger values of $N_{\gamma}$ are required in order for the semiclassical theory to hold.

We can study the behavior of the full quantum theory for different values of $N_{\gamma}$ by performing computer simulations using the MATLAB quantum optics toolbox [14]. To perform the simulations, we truncate the Fock space at 500 photons and find the steady-state solution to the master equation (2). For the simulations presented here, we choose $\eta=0.5$ and $N_{A}=0.05$; for these parameters, the semiclassical threshold is $p_{1}=0.128$ and the scaled photon number at twice the semiclassical threshold is $m\left(2 p_{1}\right)=0.918$. Graphs of $m(p)$ for different values of $N_{\gamma}$ are shown in Fig. 4. For $N_{\gamma}=50.0$, we observe a threshold that approximates the predictions of the semiclassical theory, but for the other values of $N_{\gamma}$ the threshold is completely smeared out by quantum fluctuations, and the system deviates significantly from the predictions of the semiclassical theory.

We can also use the computer simulations to investigate the correlation properties of the output light. Using the steady-state density matrix, we calculate the second-order correlation function at zero time delay,

$$
g_{2}(0)=\frac{\left\langle a^{\dagger} a^{\dagger} a a\right\rangle}{\left\langle a^{\dagger} a\right\rangle^{2}} \text {. }
$$

Figure 5 shows graphs of $g_{2}(0)$ versus pumping strength $p$ for different values of the critical photon number $N_{\gamma}$. For
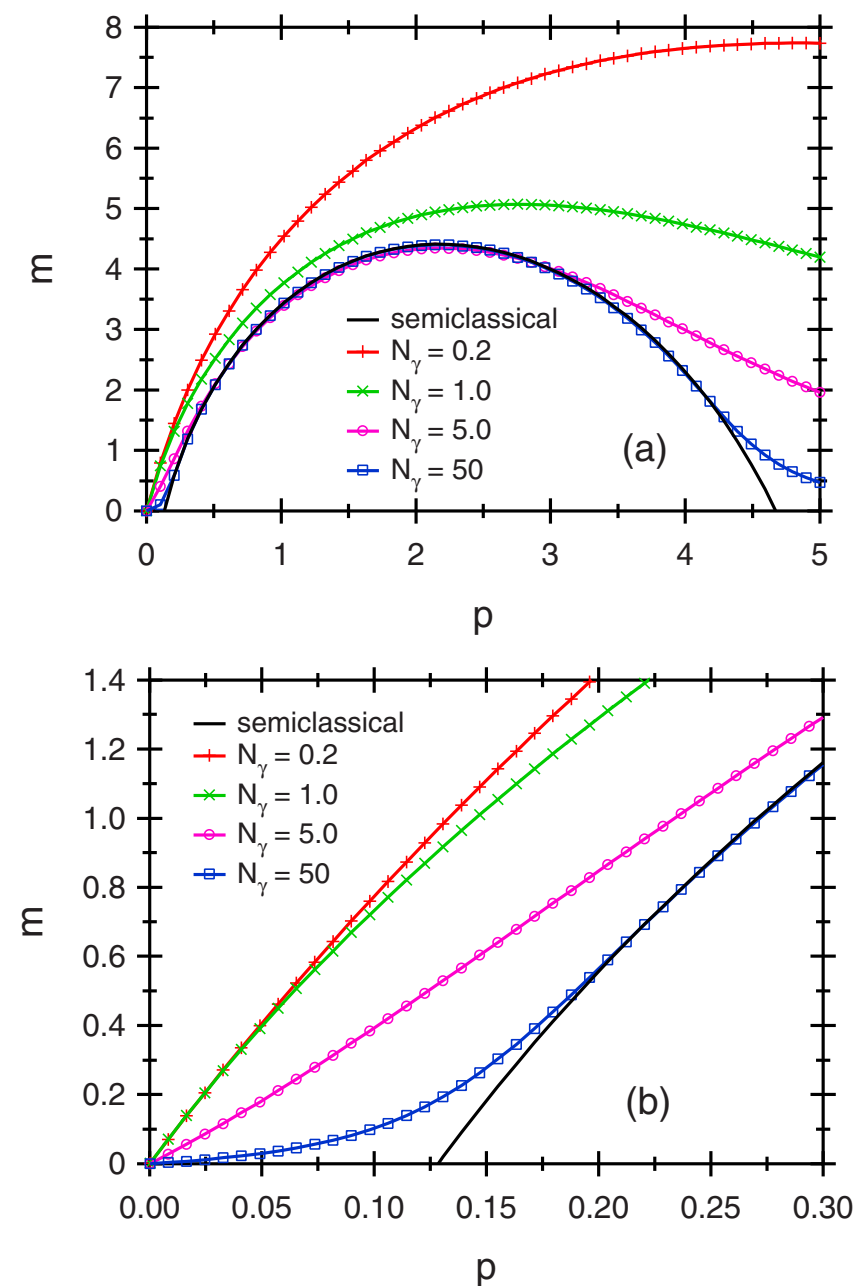

FIG. 4. (Color online) Full quantum simulation: scaled photon number $m$ versus pumping strength $p$ for $\eta=0.5, N_{A}=0.05$, and $N_{\gamma}=0.2,1.0,5.0,50.0$. In each graph, the solid curve is the semiclassical prediction given by Eq. (36).

$N_{\gamma} \gg 1$, the system behaves like a conventional laser: far below threshold the light is chaotic $\left[g_{2}(0) \simeq 2\right]$, and far above threshold the light is coherent $\left[g_{2}(0)=1\right]$. For $N_{\gamma} \ll 1$, the output light is antibunched $\left[g_{2}(0)<1\right]$ in the limit of weak driving.

\section{EFFECTIVE TWO-LEVEL ATOM}

We have seen that for large values of $N_{\gamma}$ [specifically for $\left.N_{\gamma} \gg 1 / m\left(2 p_{1}\right)\right]$, the full quantum system can be approximated using the semiclassical laser theory presented in Sec. III. We will now show that for small values of $N_{\gamma}$ (specifically for $N_{\gamma} \ll N_{A}^{2}$ ), the full quantum system can be approximated by a different theory, in which the system is treated as an effective two-level atom. In what follows, we will assume that $\left(\eta, N_{A}\right)$ lies within the semiclassical lasing regime (i.e., below the curve indicated in Fig. 2), and this assumption implies that $N_{A} \ll 1$.

We first note that from the definitions of $N_{A}$ and $N_{\gamma}$, it follows that 

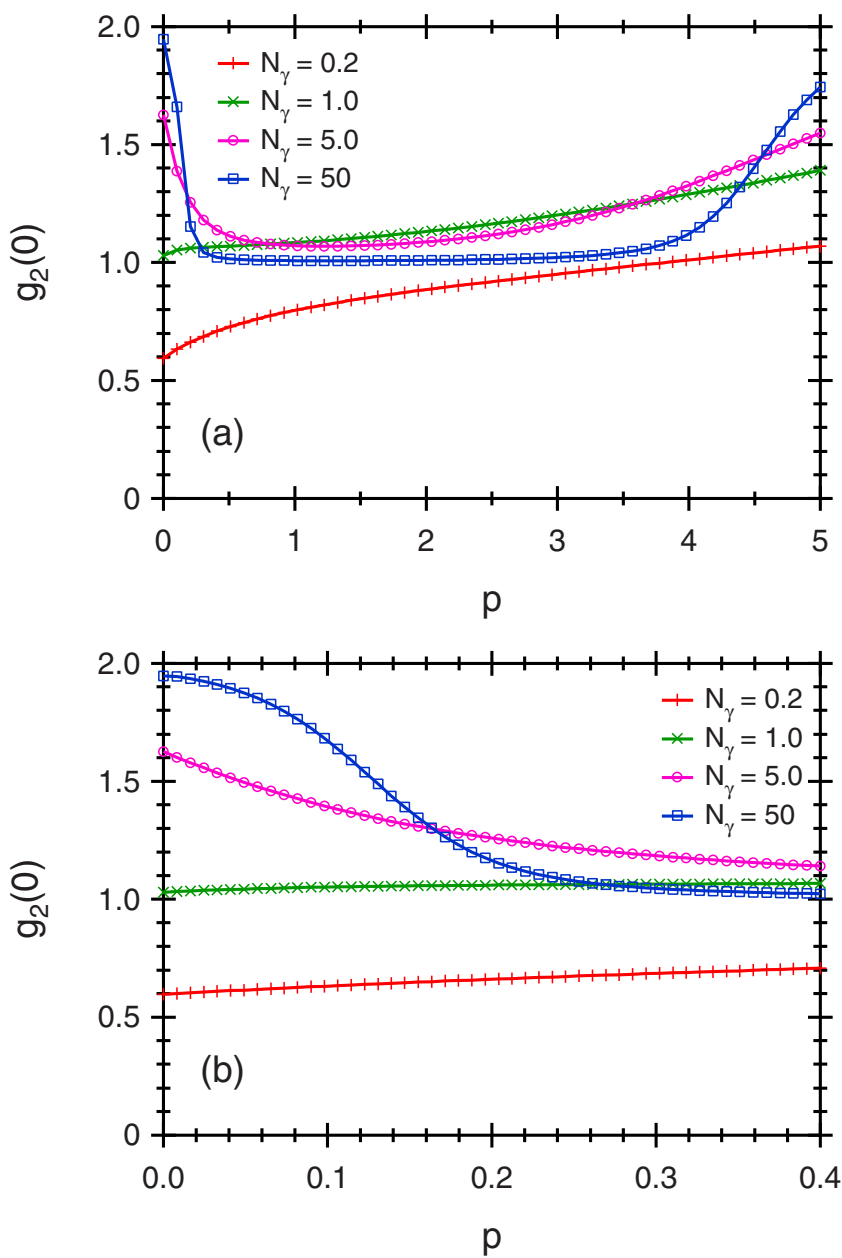

FIG. 5. (Color online) Full quantum simulation: $g_{2}(0)$ versus pumping strength $p$ for $\eta=0.5, \quad N_{A}=0.05, \quad$ and $N_{\gamma}$ $=0.2,1.0,5.0,50.0$.

$$
(2 g / \kappa)^{2}=N_{\gamma} / N_{A}^{2}
$$

Thus, if $N_{\gamma} \ll N_{A}^{2}$, then $2 g \ll \kappa$, and in this limit the coherent coupling of the 2-3 transition to the cavity mode can be approximated as an effective decay from state 3 to state 2 via the emission of a photon from the cavity, where the effective decay rate is given by

$$
\gamma_{E}=4 g^{2} / \kappa
$$

Recall that state 3 can also decay to state 2 via the spontaneous emission of a photon into free space, and this process occurs at rate $\gamma$. The ratio of these two decay rates is

$$
\gamma / \gamma_{E}=\kappa \gamma / 4 g^{2}=N_{A}
$$

Since we are assuming that $N_{A} \ll 1$, it follows that $\gamma \ll \gamma_{E}$ and therefore $\gamma$ can be neglected. The net effect of these approximations is to replace the original system shown in Fig. 1 with the simplified system shown in Fig. 6(a).

We can further simplify the system by considering the weak-driving limit in which $\Omega \ll \gamma_{E}$. Note that

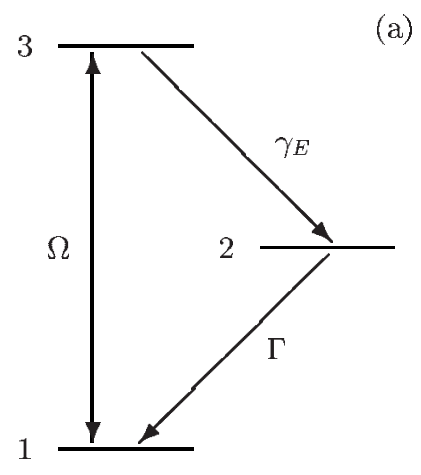

(a)

(b)

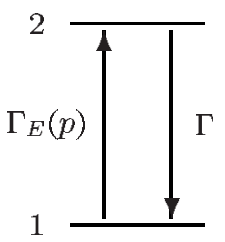

FIG. 6. Approximating the full quantum system as an effective two-level atom. (a) Step 1: replace the cavity coupling $g$ and the cavity decay $\kappa$ with an effective decay $\gamma_{E}$ form state 3 to state 2. (b) Step 2: eliminate state 3 , and replace the classical field $\Omega$ and the effective decay $\gamma_{E}$ with an effective decay $\Gamma_{E}(p)$ from state 1 to state 2 .

$$
\left(\Omega / \gamma_{E}\right)^{2}=\left(N_{A}^{2} / \eta\right) p
$$

so the weak-driving limit applies when $p \ll \eta / N_{A}^{2}$. Using Eq. (35), one can show that $p_{2} \ll \eta / N_{A}^{2}$, so the weak-driving limit applies over the entire range of pumping strengths for which the semiclassical theory predicts a nonzero laser output. In the weak-driving limit, the population in state 3 is given by

$$
P_{3}=\left(\Omega / \gamma_{E}\right)^{2}=\left(N_{A}^{2} / \eta\right) p,
$$

and the classical driving field on the 1-3 transition can be approximated as an effective decay from state 1 to state 2 , where the effective decay rate is given by

$$
\Gamma_{E}(p)=\Omega^{2} / \gamma_{E}=\Gamma N_{A} p .
$$

Since $P_{3} \ll 1$ in the weak-driving limit, state 3 can be neglected and the system reduces to an effective two-level atom, as shown in Fig. 6(b). The effective two-level atom consists of states 1 and 2, where state 2 spontaneously decays to state 1 at rate $\Gamma$ via the emission of a photon into free space, and state 1 spontaneously decays to state 2 at rate $\Gamma_{E}(p)$ via the emission of a photon from the cavity.

Using the effective two-level model, we can solve for the steady-state atomic populations $P_{1}$ and $P_{2}$ :

$$
\begin{gathered}
P_{1}=\Gamma /\left[\Gamma+\Gamma_{E}(p)\right]=\left(1+N_{A} p\right)^{-1}, \\
P_{2}=\Gamma_{E}(p) /\left[\Gamma+\Gamma_{E}(p)\right]=1-P_{1} .
\end{gathered}
$$

The steady-state photon number $n$ and scaled photon number $m$ are then given by

$$
\begin{gathered}
n=(1 / \kappa) \Gamma_{E}(p) P_{1}=\left(N_{\gamma} / \eta N_{A}\right) P_{2}, \\
m=n / N_{\gamma}=P_{2} / \eta N_{A} .
\end{gathered}
$$

Note that $P_{1}, P_{2}$, and $m$ are independent of $N_{\gamma}$. We can perform computer simulations of the full quantum system to check these predictions: graphs of $m(p)$ and $P_{2}(p)$ for different values of $N_{\gamma}$ are shown in Fig. 7. As before, we choose $\eta=0.5$ and $N_{A}=0.05$. For these parameters, the system is in the atomlike regime when $N_{\gamma} \ll N_{A}^{2}=2.5 \times 10^{-3}$, and in the weak-driving limit when $p \ll \eta / N_{A}^{2}=200.0$. 

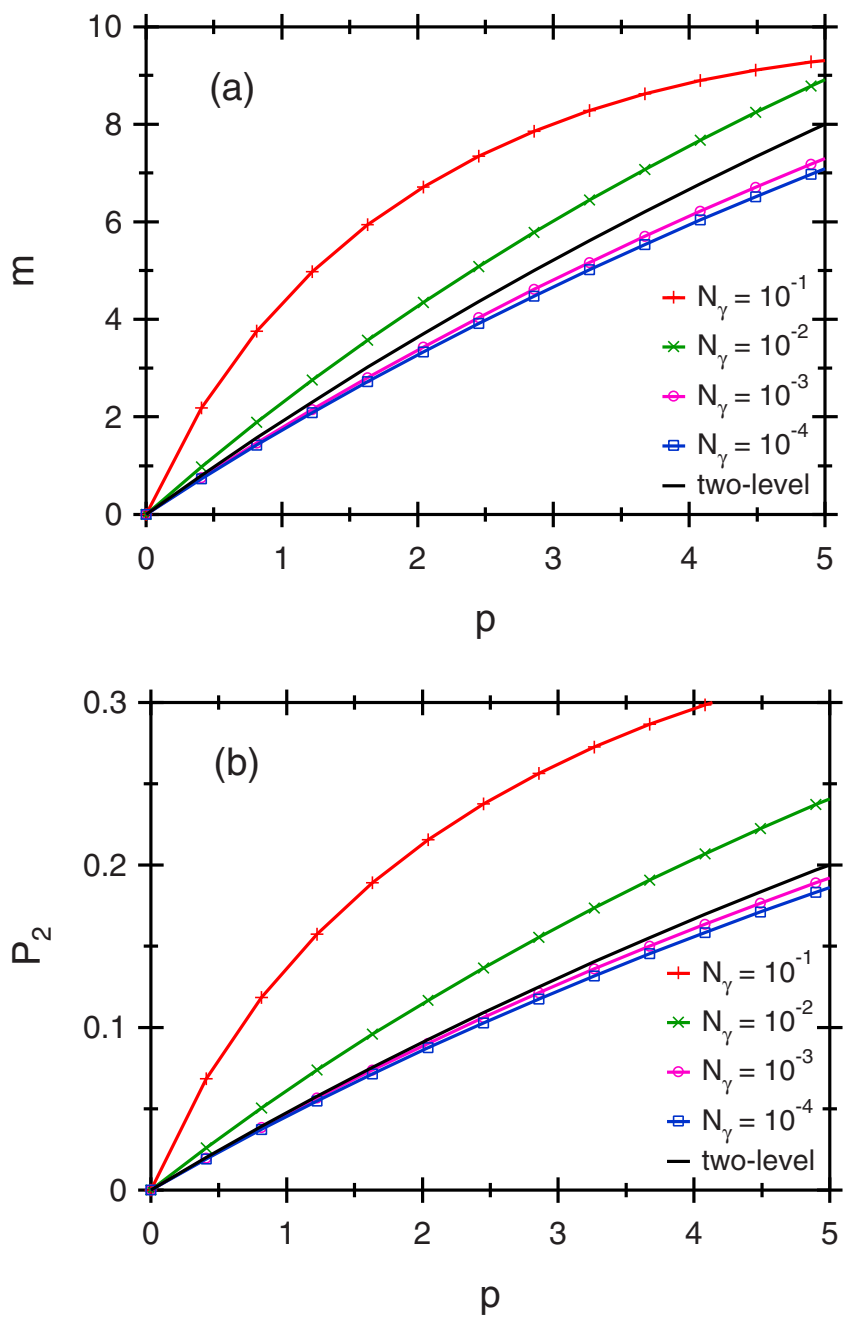

FIG. 7. (Color online) Full quantum simulation for $\eta=0.5, N_{A}$ $=0.05$, and $N_{\gamma}=10^{-1}, 10^{-2}, 10^{-3}, 10^{-4}$. (a) Scaled photon number $m$ versus pumping strength $p$. (b) Atomic population $P_{2}$ versus pumping strength $p$. The solid curves are the two-level atom predictions given by Eqs. (52) and (54).

We can use the effective two-level model to understand some of the features discussed in the previous section. From Eq. (54), we see that the semiclassical threshold is completely eliminated, and in the limit of small pumping strength the output increases linearly with pumping strength. We can also understand the antibunching of the output light: after emitting a photon from the cavity, the system is in state 2 , and it takes a time $\sim 1 / \Gamma$ until the system can return to state 1 and emit a second photon.

In summary, in the limit of small critical photon number $\left(N_{\gamma} \ll N_{A}^{2}\right)$ and weak driving $\left(p \ll \eta / N_{A}^{2}\right)$, the system acts like an effective two-level atom and the system is in the atomlike regime. In contrast, when the critical photon number is large $\left[N_{\gamma} \gg 1 / m\left(2 p_{1}\right)\right]$, the system is described by semiclassical laser theory, and the system is in the laserlike regime.

\section{CONCLUSION}

We have considered a simple model of a one-atom laser that consists of a three-level atom coupled to a mode of a high finesse cavity. We have identified two distinct parameter regimes: one in which the system behaves like a conventional laser, and one in which the system behaves like an effective two-level atom. In the laserlike regime, the system obeys semiclassical laser theory, has a well-defined laser threshold, and emits coherent light. In the atomlike regime, the semiclassical theory breaks down, there is no threshold, and the emitted light is antibunched. The model should help to clarify the meaning of the photon antibunching observed in [13], and suggests a goal for future experimental work of constructing a one-atom laser that operates in the laserlike regime.

\section{ACKNOWLEDGMENTS}

The author would like to thank A. Boca for preparing the graphs. This research was supported by the National Science Foundation, the Army Research Office, and the Disruptive Technology Office of the Department of National Intelligence.

\section{APPENDIX: ALTERNATIVE DERIVATION OF THE SEMICLASSICAL EQUATIONS OF MOTION}

In this appendix, we derive the semiclassical equations of motion (15)-(21) by replacing the full Hamiltonian $H$ with effective Hamiltonians $H_{a}$ and $H_{c}$ for the atom and cavity. Starting from the full Hamiltonian $H$, we obtain $H_{a}$ by replacing the operator $\hat{a}$ with a complex parameter $\alpha$ that represents the cavity field amplitude, and we obtain $H_{c}$ by replacing the operators $\hat{A}_{i j}$ with complex parameters $A_{i j}=A_{j i}^{*}$ that represent the atomic populations and coherences. The resulting Hamiltonians are

$$
\begin{gathered}
H_{a}=g\left(\alpha \hat{A}_{32}+\alpha^{*} \hat{A}_{23}\right)+(\Omega / 2)\left(\hat{A}_{31}+\hat{A}_{13}\right), \\
H_{c}=g\left(a A_{32}+a^{\dagger} A_{23}\right) .
\end{gathered}
$$

The atom and cavity are described by density matrices $\rho_{a}$ and $\rho_{c}$, which evolve in time according to the master equations

$$
\begin{gathered}
\dot{\rho}_{a}=-i\left[H_{a}, \rho_{a}\right]+\mathcal{L}_{12} \rho_{a}+\mathcal{L}_{23} \rho_{a}, \\
\dot{\rho}_{c}=-i\left[H_{c}, \rho_{c}\right]+\mathcal{L}_{c} \rho_{c} .
\end{gathered}
$$

We require self-consistently that

$$
\alpha=\operatorname{Tr}\left[a \rho_{c}\right], \quad A_{i j}=\operatorname{Tr}\left[\hat{A}_{i j} \rho_{a}\right] .
$$

We can obtain equations of motion for $\alpha$ and $A_{i j}$ by differentiating these equations:

$$
\dot{\alpha}=\operatorname{Tr}\left[a \dot{\rho}_{c}\right], \quad \dot{A}_{i j}=\operatorname{Tr}\left[\hat{A}_{i j} \dot{\rho}_{a}\right] .
$$

If we substitute the effective master equations (A3) and (A4) into these equations of motion, we obtain Eqs. (15)-(21). 
[1] G. M. Meyer and H. J. Briegel, Phys. Rev. A 58, 3210 (1998).

[2] B. Jones, S. Ghose, J. P. Clemens, P. R. Rice, and L. M. Pedrotti, Phys. Rev. A 60, 3267 (1999).

[3] Y. Mu and C. M. Savage, Phys. Rev. A 46, 5944 (1992).

[4] A. D. Boozer, A. Boca, J. R. Buck, J. McKeever, and H. J. Kimble, Phys. Rev. A 70, 023814 (2004).

[5] C. Ginzel, H. J. Briegel, U. Martini, B. G. Englert, and A. Schenzle, Phys. Rev. A 48, 732 (1993).

[6] T. Pellizzari and H. Ritsch, Phys. Rev. Lett. 72, 3973 (1994).

[7] T. Pellizzari and H. Ritsch, J. Mod. Opt. 41, 609 (1994).

[8] P. Horak, K. M. Gheri, and H. Ritsch, Phys. Rev. A 51, 3257 (1995).
[9] G. M. Meyer, H. J. Briegel, and H. Walther, Europhys. Lett. 37, 317 (1997).

[10] M. Löffler, G. M. Meyer, and H. Walther, Phys. Rev. A 55, 3923 (1997).

[11] G. M. Meyer, M. Löffler, and H. Walther, Phys. Rev. A 56, R1099 (1997).

[12] S. Y. Kilin and T. B. Karlovich, J. Exp. Theor. Phys. 95, 805 (2002).

[13] J. McKeever, A. Boca, A. D. Boozer, J. R. Buck, and H. J. Kimble, Nature 425, 268 (2003).

[14] S. M. Tan, J. Opt. B: Quantum Semiclassical Opt. 1, 242 (1999). 